\title{
Comprehensive Rehabilitation Program Versus Traditional Chest Physiotherapy for Weaning from Mechanical Ventilator ; randomized clinical trial
}

Sherin H. M. Mehani ( $\sim$ drsherinm@gmail.com )

Physical Therapy Department for Internal Medicine, Faculty of Physical Therapy , Beni-Suef University Hatem ELmoutaz

Anesthesia and Intensive Care Department, Faculty of Medicine, Beni- Suef University.

\section{Awny F. Rahmy}

Physical Therapy for Cardiovascular / Respiratory Disorder \& Geriatrics, Faculty of Physical Therapy, Cairo University

Ahmed M. S. Nour Eldin

Faculty of Physical Therapy, Cairo University

Ahmed Z. Fouad

Department of Anesthesia and Intensive Care, Faculty of Medicine, Cairo University.

\section{Research Article}

Keywords: Comprehensive rehabilitation, General surgery, Diffıcult weaning, Traditional chest physiotherapy

Posted Date: March 23rd, 2020

DOl: https://doi.org/10.21203/rs.3.rs-18220/v1

License: (9) This work is licensed under a Creative Commons Attribution 4.0 International License. Read Full License 


\section{Abstract}

\section{Background}

Patients needing prolonged mechanical ventilation have poor outcomes and are increasing in number. Weakness of inspiratory muscles is a well- established complication of prolonged mechanical ventilator , however the role of physical therapist has been expanded towards rehabilitation rather than inspiratory care only. The purpose of this study was to compare between comprehensive rehabilitation program and traditional chest physiotherapy on weaning from mechanical ventilation, improvement of respiratory muscle strength, peripheral muscle strength and functional status.

\section{Methods}

Fifty mechanically ventilated general surgery patients from both sexes (men \& women) with age from 40 to 50 years old participated in the present randomized clinical trial. They had difficult and prolonged weaning. They were divided randomly into two groups, $\operatorname{group}(\mathbf{A})$ consisted of twenty four patients who received comprehensive rehabilitation program combined with inspiratory muscle training and group(B) consisted of twenty six patients who received traditional chest physical therapy combined with inspiratory muscle training. Four patients in the study group and six subjects in the control group died during the intervention period and thus their data were excluded from the final analysis. Maximal inspiratory pressure (MIP), peripheral muscle strength and Functional independence measure questionnaire were measured at the beginning of the study and at weaning from mechanical ventilator.

\section{Results}

Results revealed that there was a significant improvement in days to wean and peripheral muscle strength in difficult and prolonged weaning patients in group A compared with group $B$. There was a significant improvement in maximal inspiratory pressure (MIP) in both groups. In addition, there was a significant increase in the median values of functional independence measures in group A more than group B.

\section{Conclusions}

Both physical therapy programs inspiratory muscle training and comprehensive rehabilitation and inspiratory muscle training and traditional chest physical therapy showed an increase in inspiratory muscle strength. On the other hand comprehensive program consisted of peripheral muscle training and functional training was more superior in decreasing days to wean and increasing peripheral muscle strength and functional independence measure score.

The trial was prospectively registered at Clinicaltrials.gov ; Identifier: NCT04021615 ; date : 13-7-2019

\section{Introduction}


Failure -to- wean patients represents a subset of patients receiving mechanical ventilation for a duration exceeding 15 days [1].

The ventilated patients could be categorized into three groups based on the difficulty and duration of weaning process ; simple weaning ( group 1) includes patients who pass the first weaning trial and are extubated without difficulty, difficult weaning (group 2) includes patients who fail the first trial and require up to three spontaneous breathing trials or seven days, prolonged weaning (group 3 ) includes patients who require more than seven days after the first breathing trial [2]. Although difficult- to wean patients represents about $6 \%$ of the patients inside the intensive care unit, they consume a third of resources [3] .

Failure to wean (FTW) from mechanical ventilation is a significant problem due to its economic and clinical burden [4]. The etiology of FTW could be related to weakness of the inspiratory muscles with a maximal inspiratory pressure less than $-20 \mathrm{cmH} 2 \mathrm{O}$. This will create imbalance between the pressuregenerating capacity of these muscles and the load or demand placed on them [2].

There are two types of loads that could be added to the respiratory muscles ; the elastic load resulting from the ventilator-induced dynamic hyperinflation and intrinsic positive end- expiratory pressure. The other type of load is the resistive one imposed by endotracheal tube and concrete secretions. The load could be further increased by inappropriate ventilator adjustments and patient- ventilator dyssynchrony [5].

The European Respiratory Society and European Society of Intensive Care Medicine taskforce has recommended a hierarchy of ICU mobilization based on progressively increasing exercise intensity; decubitus change and functional positioning , passive mobilization , active- assisted and active exertion , cycloegonometry in bed, sitting in bed orthostatism, static walking, transferring from bed to chair, chair exertion and walking [6].

The role of physical therapy in the intensive care unit had been expanded to be functional restoration of the patient, rather than only respiratory care [7]. Critically ill patients in ICU suffer from ventilator- induced diaphragmatic atrophy and critical illness polyneuromyopthy which usually affect the weaning , extubation , and early discharge as targeting outcomes $[8,9]$.

Chest physiotherapy may help in preventing atelectasis, removing bronchial secretions and decreasing airway resistance. These techniques includes positioning , bronchial hygiene, deep breathing exercises[10].

Targeted mobility therapy (TMT), simulating comprehensive rehabilitation program that was used in the present study, is a novel strategy for managing critically ill patients. This concept worked as a scientifically- driven, dynamic bundle of rehabilitation intervention that include inspiratory muscle strength training , bronchial hygiene, and active mobilization. This concept would guarantee the best 
outcomes for critically ill patients with difficult and prolonged weaning as regards weaning success, days on mechanical ventilator, length of hospital stay and functional mobility [7].

The evidence supporting physical therapy techniques for weaning from ventilator is very limited. On the other hand , techniques used in clinical trials were difficult making selection of the best physical therapy modalities problematic. The aim of the present study was to compare between the effect of comprehensive rehabilitation program combined with inspiratory muscle training and traditional chest physical therapy combined with inspiratory muscle training on weaning from mechanical ventilation ( days to wean and success of weaning) as primary outcomes, inspiratory muscle strength, peripheral muscle strength, functional status as secondary outcomes.

\section{Materials And Methods}

This study sample included difficult and prolonged weaning patients who fail one weaning attempt of spontaneous breathing trial and who were conscious and able to join the training program. Please note, the instrumentation images are available in the Supplemental Files section.

\section{I) Patients}

Fifty general surgery patients who were difficult and prolonged weaning patients from both sexes (men \& women) with age ranged from 40 to 50 years old in surgical intensive care unit, Kasr Al Ainy Teaching Hospital, Cairo University and Beni- Suef Teaching Hospital , Beni -Suef University.

These patients were divided randomly into two groups; group A (study group): Twenty five patients who received comprehensive rehabilitation program consist of upper-extremity exercises, lower extremity exercises, pedaling exercises, trunk control exercises and bedside functional training combined with inspiratory muscle training.

-Group B (Control group): Twenty five patients who received traditional chest physical therapy (positioning - modified postural drainage - percussion - vibration) combined with inspiratory muscle training.

A randomization process was performed using closed envelopes. The investigator prepared 50 closed envelopes, each envelope containing a card labeled either group A or group B. Each patient was asked to draw a closed envelope to determine whether he was allocated to group A or group B. The randomization (active dynamic adaptive randomization; minimization) was performed by a colleague who was independent and blind with respect to the study, using closed envelopes with the group description randomly placed within them.

The inclusion criteria was; the general surgery patients who fail one weaning attempt of spontaneous breathing trial ;adequate gas exchange ( paO2 more than $60 \mathrm{mmHg}$, $\mathrm{FiO} 2$ of 0.50 or less) conscious patients receiving assist control or SIMV of equal to or less than $6 \mathrm{~b} / \mathrm{min}$ or pressure support via trachestomy with SIMV of equal to or less than $6 \mathrm{~b} / \mathrm{min}$, pressure support of equal to or less than 15 
$\mathrm{cmH} 2 \mathrm{O}$ and PEEP of equal to or less than $10 \mathrm{cmH} 20$;no excessive secretions requiring suctions more than once every hour ; hemodynamically stable patients (lack of hypotension or a need for only low-dose pressors) ; The patients with Acute Physiology and Chronic Health Evaluation II (APACHE II) scale 20 or less.

Exclusion criteria was; patients with persistent altered sensorium ; patients with major cardiac arrhythmia ; patients with comorbid medical conditions (e.g., neurological diseases) or who are under any sedative or paralytic agents that would interfere with strength measurements and limb exercises ;patients with history of underlying neuromuscular disease or acquired polyneuromyopathies ;patients who are unable to perform physical training due to preexisting joint dysfunction ;obese patients

\section{II) Instrumentation}

\section{A- Evaluation tools and equipments}

\section{The APACHE II (Acute Physiology and Chronic Health Evaluation II)}

The Apache-II Score provides an estimate of ICU mortality based on a number of laboratory values and patient signs taking both acute and chronic disease into account [11]. It was measured by a colleague who was independent and blind with respect to the study.

\section{Mechanical ventilator}

The maximal inspiratory pressure was measured by mechanical ventilator by a colleague who was independent and blind with respect to the study .

\section{Functional Independence Measure (FIM)}

The Functional Independence Measure (FIM) is an outcome measure of the severity of disability for an inpatient rehabilitation setting. It rates 18 activities of daily living on a 7-point scale ranging from fully dependent (1) to independent with no aids (7). In this study only 5 activities were measured by a colleague who was independent and blind with respect to the study, which were bathing, dressing, moving from bed

\section{Hand-held dynamometer}

A hand-held dynamometer (Lafayette manual muscle test system Model 01163, White Plains, New York, 10602) is a portable device that can be used to obtain more discrete, objective measures of strength during manual muscle testing (MMT) than can be achieved via traditional manual muscle testing. It was measured by a colleague who was independent and blind with respect to the study

\section{B) Training equipments}

\section{Pedaling Exerciser}


A rehabilitation device (UNI-FORCE RPM26001, Profit Country Enterprises Ltd, Kowloon, Hong Kong) that allows application of various loads for both leg (pedaling) and arm (cranking).

\section{Weights}

Weights from 100 grams to 600 grams were used to progressively increase resistance.

\section{Inspiratory muscle trainer}

The patient inhaled through a device (RES-HS553-010) with an adjustable, spring-loaded, threshold poppet valve and the patient must generate an inspiratory pressure greater than the indicated threshold pressure setting to compress the spring and open the poppet valve, and the inspiratory pressure must be maintained above the threshold pressure to keep the poppet valve open.

\section{Mechanical vibrator}

The mechanical vibrator (Thrive, Model 707A, Daito electric machine industry Co., LTD, Japan) was applied to patient chest during expiration to assist airway clearance.

\section{III) Procedure}

\section{A-Evaluation procedure}

The patient was evaluated at the beginning of the study and at weaning from mechanical ventilation by a colleague who was independent and blind with respect to the study; using the following:

\section{1-Maximal inspiratory pressure}

It was conducted after endotracheal or trachestomy aspiration. Maximal inspiratory pressure was performed three times with two minutes rest in between manoeuvers. The highest value of the one second plateau pressure generated on one of the manoeuvers was considered for analysis. The mechanical ventilator was used to measure the plateau pressure (maximal inspiratory pressure). Plateau pressure was measured using the inspiratory hold function on the venilator while the patient was sedated and on volume control mode [12].

The plateau pressure was measured by ventilator in millibar then it converted to $\mathrm{cm} \mathrm{H} 2 \mathrm{O}$ by multiplying it by 1.01971621298 (1millibar equals $1.01971621298 \mathrm{~cm} \mathrm{H} 20$ and near the figure to the nearest two decimal places.

\section{2-Upper and lower extremity muscle strength}

Upper and lower extremity muscle strength were assessed using a handheld dynamometer. The shoulder flexor, elbow flexor, and knee extensor muscle groups were included in the measurements. Standard test positions was modified because all patients in this study were using mechanical ventilator and unable to sit up at initial examination. The shoulder and elbow flexors were tested in the semi-Fowler position. 
The isometric force of the shoulder flexors was tested with the shoulder flexed 90 degrees and the elbow in extended position. The dynamometer was placed just proximal to the epicondyles of the humerus, and the patients were stabilized at the axillary region. The isometric force of the elbow flexors was tested with the elbow flexed 90 degrees, the forearm supinated, and the shoulder in neutral position; the dynamometer was placed just proximal to styloid processes of ulna and radius, and the patients were stabilized at the superior aspect of the arm. All patients in the study were receiving mechanical ventilation through artificial airway; therefore, the recommended test position (i.e., prone position) for the knee extensors was modified to the supine position, with the knee slightly flexed at 20 to 50 degrees, a roller behind the knee, the ankle not touching the bed, and the hands resting on the lap. The dynamometer was placed just proximal to the malleoli of the tibia and fibula. The dynamometer shaft was held perpendicular to the tested limb segment, and the tester applying all manual stabilization. All measurements were performed 3 times using isometric "make" tests. The patients were asked to increase force to a maximum effort over a 2-second period, maintain the maximum effort for approximately 5 seconds, and then stop. This procedure had been shown to yield reliable measurements and to be adequate for measuring maximum isometric strength. The peak force in kilogram of 3 tests was recorded. Two minutes of rest was allowed between repeated readings [13].

\section{3- The Functional Independence Measure (FIM)}

Five activities with the Functional Independence Measure (FIM) were measured, which were bathing, dressing, moving from bed to chair, bowel management, and bladder management.

\section{B-Treatment procedure}

\section{- Study group treatment procedure}

\section{1-Spontaneous breathing trials}

Spontaneous breathing trials was used when underlying cause of acute respiratory failure stabilizes or significantly improved and daily assessment of the patient's readiness for ventilator discontinuation was considered. The spontaneous breathing trial lasted from 30 minutes to 120 minutes combined with CPAP and if the trial had failed, the patient was returned back to full ventilatory support for at least 24 hours before performing a new trial [14].

The following guidelines were used when determining weaning readiness; resolution of the disease for which the patient was intubated; paO2/FiO2 of 150-200 requiring PEEP of equal to or less than 5$8 \mathrm{cmH} 2 \mathrm{O}$ and $\mathrm{FiO} 2$ of equal to or less than $0.4-0.5$; haemodynamic stability ; absence of fever ; stable blood pressure; no or minimal vasopressor ; adequate hemoglobin ; adequate mentation ; adequate cough [4].

\section{2-Inspiratory muscle training}


A threshold inspiratory muscle trainer was connected to the artificial airway with $15 \mathrm{~mm}$ and $22 \mathrm{~mm}$ adaptors and the patient exhaled through the training device via a low-resistance, one-way, spring-loaded valve through the inhalation orifice. Training bouts consisted of three to five sets of six repetitions breathing through the trainer. Inspiratory muscle strength training sessions was conducted in the morning, 5 to 7 days per week and supplemental oxygen was added as needed during training. Training was conducted with the patient in bed with an approximately $30^{\circ}$ head-up tilt and with intensity about 40 $\%-50 \%$ of maximal inspiratory pressure. Patients were instructed to maximally exhale before taking a breath and to try to maximally fill the lungs fully with each inspiration. This training breath was repeated six times in each training set. Patients were returned to mechanical ventilation for rest between training sets as needed. Three to five sets of six training breaths were completed for a total of 18 to 30 training breaths per session. Following each training set, the patient indicated a rating of perceived inspiratory exertion on modified Borg scale of 0 to 10, with 0 representing no inspiratory effort and 10 equaling a maximal inspiratory effort. The training threshold pressure setting was adjusted to an exertion rating of 6 to 8 . If an exertion rating fell below 6 , the pressure was increased, and if the exertion rating exceeded 8 , the pressure was reduced. Patients were observed during training for cardiac arrhythmias, for pulse oximetric saturation decreases of more than $5 \%$ from baseline, for significant changes in BP, and for subjective evidence of distress. A signal was used by the patient to indicate that he want to stop training before competing six repetitions and return to mechanical ventilation. The complete inspiratory muscle strength training session was about10 min per day [15] .

\section{3- Upper extremity exercises}

Upper-extremity exercises included range of motion (ROM) exercises for the wrist; elbow and shoulder flexion and extension; and shoulder abduction, adduction, and internal and external rotation, with 10 repetitions of each motion per set for 2 sets. Patients initially performed these exercises against gravity in a supine position and progressed to a sitting position as tolerated. These exercises' resistance then increased to repetitions against resistance using weights (100-600 g)

\section{4-Lower extremity exercises}

Lower extremity exercises included ROM exercises for ankle dorsiflexion and plantar flexion, hip and knee flexion and extension, and straight leg raising, with 10 repetitions of each motion per set for 2 sets in the supine position.

\section{5-Pedaling exerciser}

The patients were instructed to pedal by lower limb and arm cranking and to maintain at least 30 revolutions per minute. The training intensity and duration was progressively increased depending on the patient's ability (up to an arbitrary limit of 30 min 2 sessions /day) [16].

\section{6-Trunk control exercises}


The patients received exercises consisting of task-specific movements of the upper and lower part of the trunk both in the supine and sitting positions. The supine exercises involved the pelvic bridge, the unilateral bridge, the flexion rotation of the upper and lower trunk. Sitting exercises included selective flexion extension of the lower trunk; lateral flexion of the upper and lower trunk; rotation of the upper and the lower trunk; weight shifts; forward and lateral reach, with 10 repetitions of each motion per set for 2 sets. The trunk exercises were initiated with moderate assistance and progressed to a state of no assistance. The number of repetitions and intensity of the exercise was determined according to the patient's performance with adequate rest periods in between.

The intensity of the exercises was increased by introducing one or several of the following changes; reducing the base of support ;increasing the lever arm ; advancing the balance limits ; Increasing the hold time

\section{7-Bedside functional retraining}

Bedside functional retraining included turning from side to side on the bed; transfers to and from the bed, chair, and wheelchair; and coming to a standing position. Ambulation was begun as early as patients can tolerate it. Patients were allowed to rest between training sets, and decreased pulse oxygen saturation (SPO2) and any sign or symptom that indicate intolerance was closely monitored throughout the training session. Control group treatment procedure included inspiratory muscle training, manual percussion , mechanical vibration to increase mucociliary clearance, positioning and modified postural drainage such as high side lying or side lying.

\section{Primary outcomes}

Days to wean and success of weaning from mechanical ventilator were evaluated at weaning period

\section{Secondary outcomes}

Maximal inspiratory pressure, peripheral muscle strength and Functional independence measure questionnaire were measured at the beginning of the study and at weaning from mechanical ventilator.

\section{Statistical analysis and sample size calculation}

All these data were analyzed statistically by using:

1-Descriptive statistics (mean - standard deviation-median).

2- Inferential statistics using paired and unpaired t-test.

The level of significance was $(P<0.05)$.

3-Non-parametric statistical test for functional independence measure questionnaire (FIM). MannWhitney test between groups and Wilcoxon signed-rank test inside each group. 
Sample size calculation was performed at the beginning of the study using G_Power statistical software (version 3.1.9.2; Franz Faul, Universit€at Kiel, Germany) (Faul et al., 2009) and this revealed that the appropriate sample size for this study was $\mathrm{N}=40$ which gave an observed power equal to 0.87 . Calculations were performed using alpha $=0.05, \beta=0.2$ and an effect size of 0.6 .

\section{Results}

\section{Demographic and clinical characteristics of patients}

The demographic and clinical characteristics of the patients are shown in Table 1. At baseline, there were no statistical significant differences between both groups as regards to age, weight, height, APACHE II, MIP, right and left shoulder flexors and elbow flexors, right and left knee extensors, median values of functional independence measures for bathing, dressing, moving from bed to chair, bladder management, bowel management $(p=0.59,0.54,0.71,0.85,0.18,0.97,0.74,0.95,0.69,0.91,0.86$, $0.52,0.52,1,0.55,0.31$, respectively), see Tables $1-5$

\section{Dropout and clinical events}

Four patients in the study group and six patients in the control group died during the intervention period and thus their data were excluded from the final analysis, see Fig. 1

\section{Results of group A}

There was a statistical significant increase of MIP, right and left shoulder flexors and elbow flexors, left and right knee extensors, median values of functional independence measures $(p=0.0001,0.0001$, $0.0001,0.0001,0.0001,0.0001,0.0001,0.0001,0.0001,0.0001,0.0001,0.0001$, respectively), see Tables $2,3$.

\section{Results of group B}

There was a statistical significant increase of MIP, right and left shoulder flexors and elbow flexors, left and right knee extensors, median values of functional independence measures for bathing, dressing and bladder management only $(p=0.0001,0.0001,0.0001,0.0001,0.0001,0.0001,0.0001,0.003,0.003$, $0.31,0.003,0.31$, respectively) ), see Tables 4,5 .

\section{Comparison between group $A$ and group $B$ post training}

There was a statistical significant difference between both groups in favor of group A as regards to days to wean, right and left shoulder flexors and elbow flexors, left and right knee extensors, median values of all functional independence measures $(p=0.0001,0.0001,0.0001,0.0001,0.0001,0.0001,0.0001$, $, 0.0001,0.0001,0.0001,0.0001,0.0001$, respectively. There was a statistical non significant difference between both groups as regards to MIP, weaning success( $p=0.79,0.57$, respectively) see Tables 6,7 , Fig.2. 


\section{Discussion}

Inspiratory muscle training conducted in the rehabilitation program for both groups may have assisted the rate of successful weaning from mechanical ventilator by several mechanisms. The first explanation is reversal of disuse atrophy of respiratory muscles. A second mechanism may be altered neuromuscular dysfunction specific to inspiration as mechanical ventilator could alter the function of central and peripheral neuromuscular inspiratory components. A third possible mechanism induced by inspiratory strength training may be shifting of breathing pattern towards a more deep and slow one. When the patient inspired against about $40-50 \%$ of the maximal inspiratory pressure, more force production was achieved which led to a standardized training breath for the duration of their inspiration [17].

Inspiratory muscle training conducted in this study was dependent on threshold loading, so the patient must generate a preset pressure to allow airflow for each breath. The inspiratory flow in this type of training is not dependent on the patient effort. This means that threshold training is the easiest to prescribe and most standardized for ICU patients [18].

ICU patients usually have weak inspiratory muscles as observed in the present study, maximal inspiratory pressure was about $27 \mathrm{cmH} 2 \mathrm{O}$. Using inspiratory training protocol with high intensity and low repetitions in the present study was supported by Nava and Fasano ,2011[1] , Condessa et al. , 2013 [19].

The results of the present study was supported by Martin et al ., 2011[4] who investigated the effect of inspiratory muscle strength training with intensity set to the highest possible pressure that could be tolerated by the patient and was progressed daily. The study concluded an improvement in maximal inspiratory pressure and weaning rate in tracheostomized patients. The study attributed the results to correction of feedback discreoancy between respiratory drive and lung / chest expansion by strengthening inspiratory muscles and gaining lower perception of breathing effort.

The significant improvement in days to wean that was observed in favor of the study group who received the comprehensive rehabilitation program was coincided by Perme et al. ,2009 [20] who concluded that even high intensity exercises done in bed do not overcome the side effects of prolonged bed rest as a result of shifting of intravascular fluid away from extremities towards thoracic cavity. Practicing early mobility can help in optimal fluid distribution and improve orthostatic tolerance.

Early mobility could enhance functional outcomes as reported in the present study, there was an improvement in FIM score for bathing, dressing , moving from bed to chair, bowel and bladder management.

Evidence from systemic reviews and meta- analysis suggested that active mobilization improves the rate of successful weaning and reduces the days required to wean [7]. 
The significant effects of comprehensive rehabilitation program go ahead with Clini et al ., 2011 [21] who revealed that the program induced significant improvements in weaning success, but the rate of successful weaning was less than observed in the present study, that may be attributed to the study of Clini et al., used comprehensive rehabilitation without addition of inspiratory muscle training .

Bissett et al., 2007[22] concluded more days (27) wean patients from mechanical ventilation after laparatomy. The training program consisted of only inspiratory muscle training with an intensity between 6-8 using modified Borg scale, without including any mobilizing or functional activities.

The results of the present study showed that patients in the control group remained completely dependent, while $90 \%$ of the patients in the study group ( 18 out of 20) moved from being completely dependent to being partially dependent according to FIM score. This finding was supported by Montagnani et al ., 2011 [23] who examined the effect of progressive mobilization exercises combined with progressively decreasing the level of pressure- support ventilation or progressively longer spontaneous breathing trials.

The results of Yang et al. ,2010 [24] matches with the present study which sowed a non significant differences between both groups as regards the rate of successful weaning that was lower in that reported by Yang et al., as their study did not include threshold inspiratory training. They used traditional abdominal training and inspiratory muscle weight training combined with mobilization training.

Caruso et al., 2005 [25] applied different inspiratory muscle training protocol by adjusting the pressure trigger sensitivity for a maximum of $40 \%$ as showed a lower rate of successful weaning as contrary to the present study inspiratory muscle training protocol and results.

The higher successful weaning rate and the less days to wean from mechanical ventilator in favor of the study group may be the result of comprehensive training-induced functional independence and increased inspiratory muscle strength.

Strengthening of inspiratory muscles conducted in both training groups may explain the non significant difference in the rate of successful weaning between both groups. Inspiratory muscle strength is inversely proportional to inspiratory effort and inspiratory drive whose reduction along with increased maximal inspiratory pressure could account for successful weaning observed in the present study.

The present study was limited by only five activities of functional independence were measures ( breathing , bathing, moving from bed to chair, bowel and bladder management). Although the present study had been strengthened by being multi-centered design which augment the external validity of the collected data, these results were only applicable to post surgical patients only.

\section{Conclusion}

Both physical therapy programs; comprehensive rehabilitation program (peripheral muscle training and functional training) combined with inspiratory muscle training and traditional chest physical therapy 
(percussion, mechanical vibration, positioning and modified postural drainage) combined with inspiratory muscle training showed an increase in inspiratory muscle strength. On the other hand comprehensive program was more superior in decreasing days to wean and increasing peripheral muscle strength and functional independence measure score.

\section{Abbreviations}

\begin{tabular}{ll} 
Acute physiology and chronic health evaluation & APACHE \\
\hline Continuous positive airway pressure & CPAP \\
\hline Centimeter water & $\mathrm{CmH} 2 \mathrm{O}$ \\
\hline Functional independence measure & FIM \\
\hline Fraction of inspired oxygen & FiO2 \\
\hline Failure to wean & FTW \\
\hline Intensive care unit & ICU \\
\hline Maximal inspiratory pressure & MIP \\
\hline Millimeter mercury & mmHg \\
\hline Positive end expiratory pressure & PEEP \\
\hline Range of motion & ROM \\
\hline Synchronized intermittent mandatory ventilation & SIMV \\
\hline Targeted mobility therapy & TMT
\end{tabular}

\section{Declarations}

\section{Availability of data and materials}

The datasets used and/or analyzed during the current study are available from the corresponding author on reasonable request.

\section{Acknowledgements}

Not applicable

\section{Funding}

No funding

\section{Author information}




\section{Affiliations}

Sherin H. M. Mehani ${ }^{1}$, Hatem ELmoutaz ${ }^{2}$, Awny F. Rahmy ${ }^{3}$, Ahmed M. S. Nour Eldin ${ }^{4}$, Ahmed Z. Fouad 5

(1) Professor and Chairman of Physical Therapy Department for Internal Medicine, Vice Dean for Education and Student Affairs, Faculty of Physical Therapy , Beni-Suef University.

(2)Professor and Chairman of Anesthesia and Intensive Care Department , Faculty of Medicine, BeniSuef University.

(3)Professor of Physical Therapy for Cardiovascular /Respiratory Disorder \& Geriatrics, Faculty of Physical Therapy, Cairo University

(4) MSc in Physical Therapy, Cairo University

(5) Assistant professor of Anesthesia and Intensive Care, Faculty of Medicine, Cairo University.

\section{Contributions}

SH helped in the study design, acquisition of data, and drafting the manuscript. HE was responsible for conception of the idea, study design, analysis of the data, and drafting of the first manuscript. AF was responsible for analysis and interpretation of the data, writing the manuscript, and responding to the reviewer comments. AS, $\mathrm{HE}$ and $\mathrm{AF}$ helped in the acquisition of data, management of the patients, and revising the manuscript. $\mathrm{SH}$ is the senior author who was responsible for supervision of the whole research and revising the final manuscript. All authors approved the manuscript and agreed to be accountable for all aspects of the work.

\section{Corresponding author}

Correspondence to Sherin H. M. Mehani

\section{Ethics declarations}

\section{Ethical approval and consent to participate}

The study was approved from Faculty of Medicine, Beni - Suef University Research Ethical Committee (FMBSUREC/05032019/ELMoutaz ), written informed consent was obtained from the patients or their legally designated surrogated .

\section{Consent for publication}

Not applicable

\section{Competing interests}


The authors declare that they have no competing interests.

\section{References}

1- Nava S, and Fasano L I. Inspiratory muscle training in difficult to wean patients: work it harder, make it better, do it faster, makes us stronger . Critical Care. $2011 ; 15: 1-3$.

2- Navalesi $P$, Frigerio $P$, Patzlaff $A$, et al. Prolonged weaning : from intensive care to home. Rev Port Pneumol. $2014 ; 20$ (5) : $264-72$.

3- Jackson M , Strang T, and Rajalingam Y . A practical approach to the difficult- to- wean patients .J I C S . $2012 ; 13(4)$ : 327-31.

4- Martin A D, Smith B K, Davenport P, et al. Inspiratory muscle strength improves weaning outcomes in failure to wean patients : A randomizes clinical trail. Critical Care. 2011; 15 :R84:1-12.

5- Maclntyre NR, Epstein SK, Carson S, Scheinhorn D, Christopher K, Muldoon S, National Association for Medical Direction of Respiratory Care. Management of patients requiring prolonged mechanical ventilation: report of a NAMDRC consensus conference. Chest. 2005;128:3937-54.

6- Gosselink R, Bott J, Johnson M, Dean E, Nava S, Norrenberg M, et al. Physiotherapy for adult patients with critical illness: recommendations of the European Respiratory Society and European Society of Intensive Care Medicine Task Force on Physiotherapy for Critically III Patients. Intensive Care Med. 2008;34(7):1188-99.

7- Bhat $A$ Vasanthan $L T$, and Babu A S . Role of physiotherapy in weaning of patients from mechanical ventilation in the intensive care unit . Indian Journal of Respiratory Care. 2017 ; 6 (2) : 813-19.

8- Stiller k. Physiotherapy in intensive care : An updated systematic review . Chest .2013;144: 825-47.

9- Dres M , Dube B P, Mayaux J, et al . Coexistence and impact of limb muscles and diaphragm weakness at time of liberation from mechanical intensive care unit patients . Respiratoru Crit Care Med. $2017 ; 195: 57-66$.

10- Clini M and Ambrosino N. Early physiotherapy in the respiratory intensive care unit. Respir Med .2005;99:1096-104.

11-Knaus WA, Draper EA, Wagner DP and Zimmerman JE. APACHE II: a severity of disease classification system. Crit Care Med. 1985; 13:818-29.

12- Sidebotham D, McKee A, Gillham M and Levy J .Cardiothoracic Critical Care. In ButterworthHeinemann, Philadelphia, Pa. 2007; p.423. 
13- Chiang LL, Wang LY, Wu CP, Wu HD and Wu YT. Effects of physical training on functional status in patients with prolonged mechanical ventilation. Phys Ther. 2006; 86:1271- 81.

14- Molina- Saldarriaga FJ, Fonseca- Ruiz NJ, Cuesta-Castro PD

and Esteban A. Spontaneous breathing trial in chronic obstructive pulmonary disease: Continuous positive airway pressure ( CPAP) versus T- piece. Med Intensiva. 2010;34 (7): 453-8.

15- Martin AD, Davenport PD, Franceschi AC and Harman E. Use of inspiratory muscle strength training to facilitate ventilator weaning: a series of 10 consecutive patients. Chest. 2002; 122:192- 6.

16- Clini E, Crisafulli E, Antoni FD, Beneventi C, et al. Functional Recovery Following Physical Training in Tracheotomized and Chronically Ventilated Patients. Respir Care. 2011; 56(3):306-13.

17- Hopkins PD and Sprague SS. Use of inspiratory strength training to wean six patients who were ventilator- dependent . Phys Ther .2003;83 (2): 171-81.

18- Bissett B , Leditschke I A , Green M ,et al .Inspiratory muscle training for intensive care patients : A multidisciplinary practical guide for clinicians . Australian Critical Care. 2018:1-7 .

19- Condessa RL , Brauner JS, Saul AL , Baptista M , et al. Inspiratory muscle training did not accelerate weaning from mechanical ventilation but did improve tidal volume and maximal respiratory: a randomized trial. J Physio Ther. 2013; 59 (2):101-7.

20- Perme $C$ and Chandrashekar R. Early mobility and walking program for patients in intensive care unit: creating a standard of care. AJCC. 2009; 18 (3): 212-20.

21- Clini E, Crisafulli E, Antoni FD, Beneventi C, et al. Functional Recovery Following Physical Training in Tracheotomized and Chronically Ventilated Patients. Respir Care. 2011; 56(3):306-13.

22-Bissett B and Leditschke IA. Inspiratory muscle training to enhance weaning from mechanical ventilation. Anaesth Intensive Care .2007;25:776-9.

23- Montagnani G, Vagheggini G, Vlad EP, Berrighi D, et al.. Use of the Functional Independence Measure in People for Whom Weaning From Mechanical Ventilation Is Difficult. Phys Ther. 2011; 91:1109-15.

24-Yang PH and Wang CS. Outcome of Physical Therapy Intervention on Ventilator Weaning and Functional Status. Kaohsiung J Med Sci. 2010; 26:366-72.

25- Caruso P, Denari SD and Ruiz SA. Inspiratory muscle training is ineffective in mechanically ventilated critically ill patients. Clinics. 2005; 60:479- 84.

\section{Tables}

Due to technical limitations, the tables are only available as a download in the supplemental files section. 


\section{Figures}

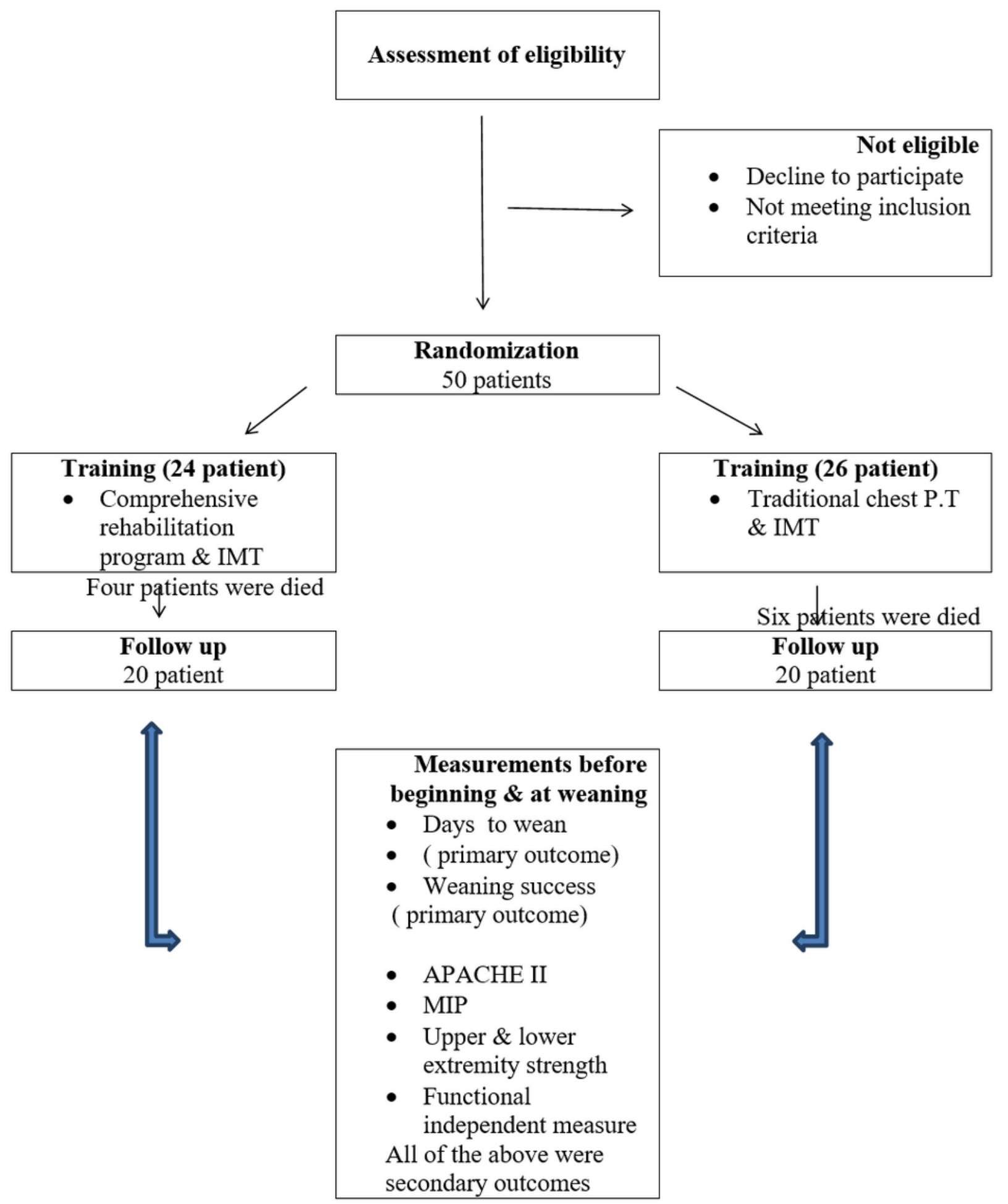

Figure 1

Flow diagram of the participants throughout the study 


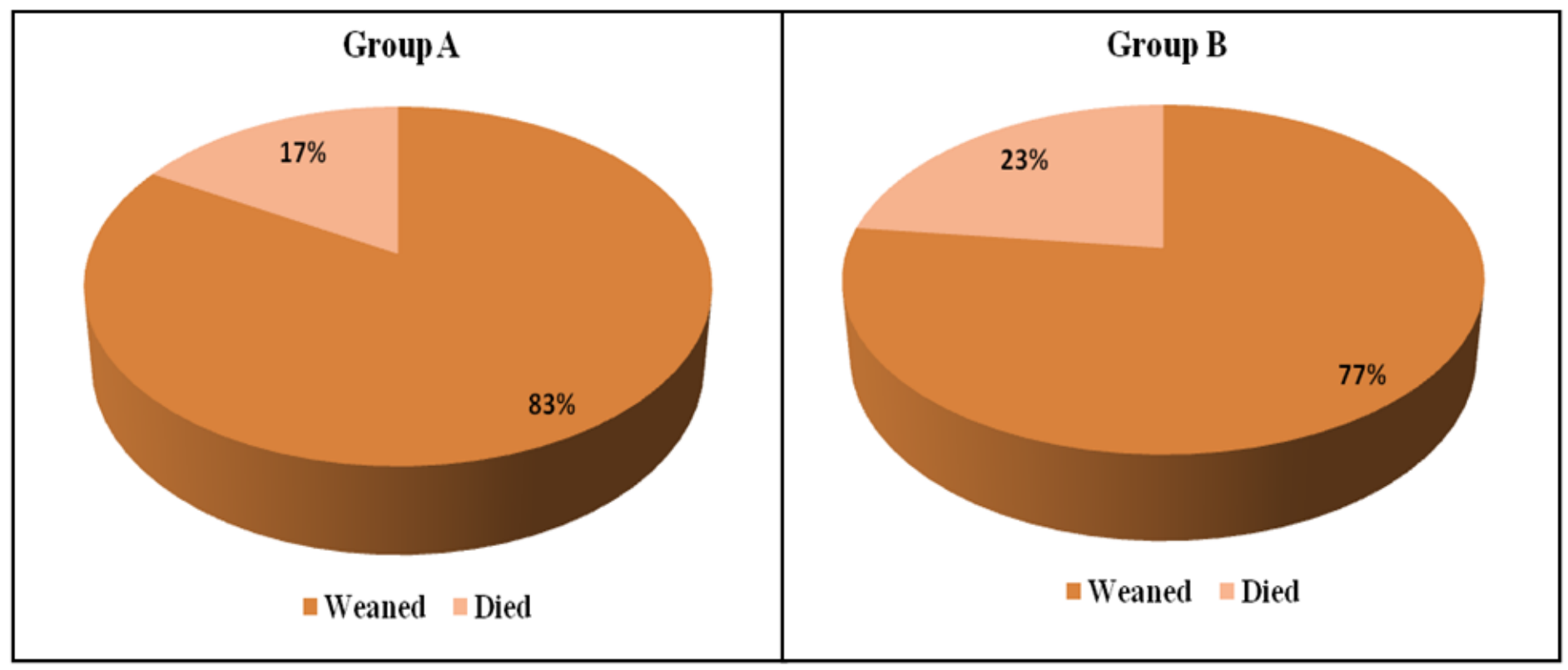

Figure 2

The frequency distribution and chi squared test for comparison of percentage of successful weaning in both groups $(A$ and $B)$

\section{Supplementary Files}

This is a list of supplementary files associated with this preprint. Click to download.

- Tables.pdf

- Instrumentationwithimages.pdf 